\title{
Transient Osteoporosis of Hip with Bone Marrow Edema Syndrome in a Pregnant Woman in Tirunelveli, Tamil Nadu, India
}

\author{
Sumathi Chellapa ${ }^{1}$, Venkatesh Babu Subbiah ${ }^{2}$
}

\begin{abstract}
Case description: After normal delivery of her healthy child 20 days back, this 30-year-old woman presented to the authors with significant left hip pain and radiation to her left thigh limiting her routine activities. We are presenting the rare condition of transient osteoporosis of hip (TOH) with bone marrow edema which had been successfully managed with conservative treatment in the southern region of India.

Conclusion: We validate that our Indian pregnant women can face the challenge of this rare condition of TOH with bone marrow edema which can be treated well.

Keywords: Bone marrow edema Syndrome, Hip, Osteoporosis, Pregnancy, Transient.

Journal of Orthopedics and Joint Surgery (2020): 10.5005/.jp-journals-10079-1016
\end{abstract}

\section{INTRODUCTION}

Transient osteoporosis of hip (TOH), or transient bone marrow edema syndrome (TBME), of unknown pathogenesis been reported well in the Indian subcontinent since 2009. ${ }^{1}$ This rare condition occurs in middle-aged men and younger pregnant women in their third trimester. ${ }^{1-4}$ It had occurred in a 50 -year-old man as well in India ${ }^{4}$

We present the case of primigravida with TOH/TBME of left hip and well treated in the developing city of the Tamil Nadu State in India.

\section{Case Description}

We are presenting a young woman who had been suffering with $\mathrm{TOH}$ and effectively managed. We authorize that our Indian pregnant women can get this rare condition of $\mathrm{TOH}$ with bone marrow edema. When diagnosed early, it can be treated well (Figs 1 to 3 ).

After normal delivery of her healthy child 20 days back, this 30-year-old woman presented to the authors with significant left hip pain and radiation to her left thigh limiting her routine activities. The pain started in the third trimester, but it was ignored and treatment was delayed. However, she pursued our conservative therapy with bisphosphonates and calcium (risedronate with calcium citrate maleate and cholecalciferol) for three months and recovered to her normal activities in this period. We are presenting this rare condition of $\mathrm{TOH}$ with bone marrow edema which had been successfully managed in the southern region of India.

\section{Discussion}

Indian subcontinent had witnessed many pregnant women and middle-aged men are suffering from this disease in the recent two decades. ${ }^{5}$ In New Delhi there was a study had high incidence of men getting involved with $\mathrm{TOH}^{6}{ }^{6}$

Since it was first reported, it is a disease of the pregnant women well diagnosed clinically and radiologically through the
${ }^{1}$ Department of Obstetrics and Gynaecology, Sri Sakthi Hospital, Tirunelveli, Tamil Nadu, India

${ }^{2}$ Department of Orthopaedics and Trauma Surgery, Sri Sakthi Hospital, Tirunelveli, Tamil Nadu, India

Corresponding Author: Venkatesh Babu Subbiah, Department of Orthopaedics and Trauma Surgery, Sri Sakthi Hospital, Tirunelveli, Tamil Nadu, India, Phone: +91 9843057118, e-mail: drsvbabu@hotmail. com

How to cite this article: Chellapa S, Subbiah VB. Transient Osteoporosis of Hip with Bone Marrow Edema Syndrome in a Pregnant Woman in Tirunelveli, Tamil Nadu, India. J Orth Joint Surg 2020;2(1):41-42.

Source of support: Nil

Conflict of interest: None

magnetic resonance imaging (MRI). This condition produces severe hip pain and does limit normal activities. With a high index of clinical suspicion, MRI is the key element in diagnosing

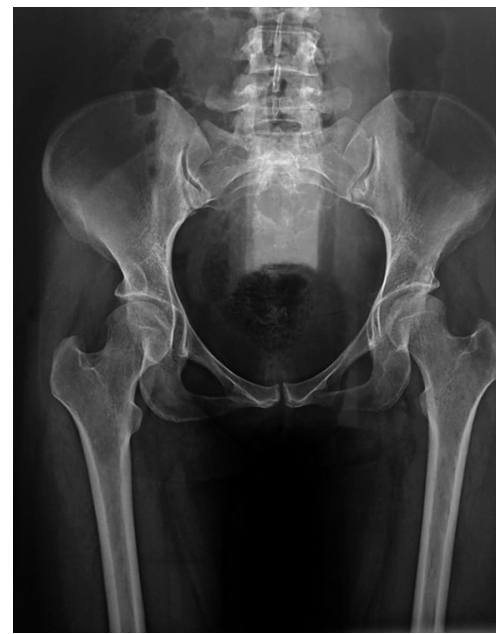

Fig. 1:'Normal'X-ray pelvis

(0) The Author(s). 2020 Open Access This article is distributed under the terms of the Creative Commons Attribution 4.0 International License (https://creativecommons. org/licenses/by-nc/4.0/), which permits unrestricted use, distribution, and non-commercial reproduction in any medium, provided you give appropriate credit to the original author(s) and the source, provide a link to the Creative Commons license, and indicate if changes were made. The Creative Commons Public Domain Dedication waiver (http://creativecommons.org/publicdomain/zero/1.0/) applies to the data made available in this article, unless otherwise stated. 


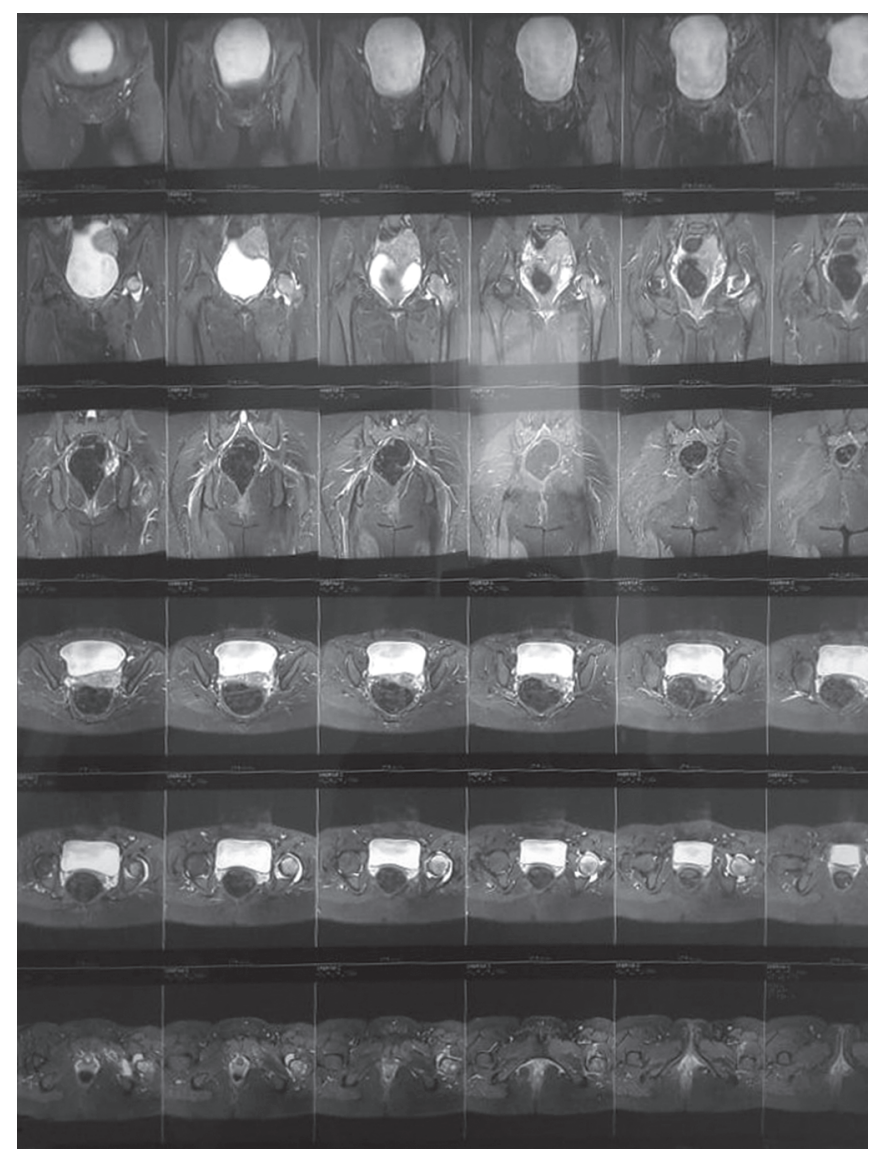

Fig. 2: Magnetic resonance imaging showed left transient osteoporosis of hip

the disease. It causes effusion of hip joint and surrounding soft tissues. The conservative line of management including strict bed rest, analgesics with oral bisphosphonates completely cures the disease in a few months. The condition may produce stress fracture of the proximal femur if it is ignored and severe physical exhaustion is caused. ${ }^{7}$

The presentation in the hip is dull aching pain in the hip progressing over a period of time, with terminal limitation of movements. As classically reported, our patient had her hip getting involved but there are reports of involvement of spine, knee, ankle, and foot. ${ }^{6,8}$ The TOH may have migratory features. Both regional migratory osteoporosis (RMO) and $\mathrm{TOH}$ are considered part of the same spectrum. However, RMO involves the hip less commonly. It principally involves ankle, foot, and knee. ${ }^{1}$

Preliminary X-rays are most of the times normal in these patients, because it takes time for the osteopenia to develop. Typical radiographic findings are present within 4-8 weeks after onset of symptoms. But MRI is the proven diagnostic and rules out other causes of hip pain in the adult. Also, MRI shows distinctive features of bone marrow edema as early as 48 hours following the onset of pain. Hence it is better to take the MRI along with X-ray if we suspect the TOH clinically. ${ }^{1}$

As per the evidence, we advised her protective weight bearing. Oral bisphosphonates and calcium were given to her with regular follow-up.

Though the hip effusion is not so common, it was also seen in our case.

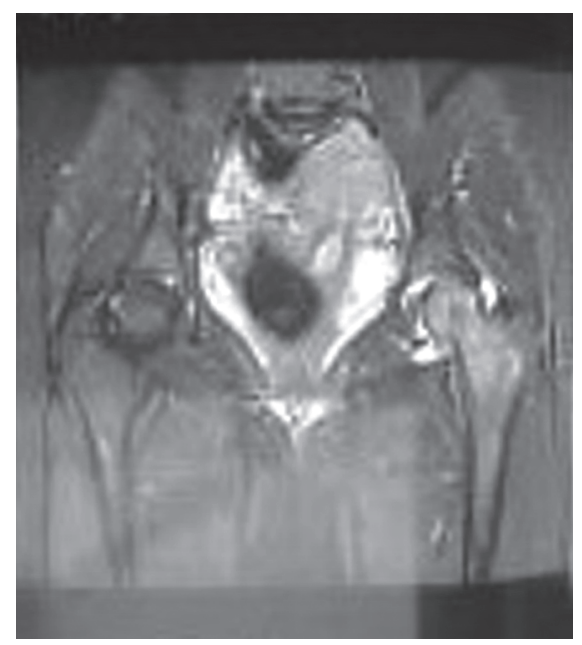

Fig. 3: Magnetic resonance imaging picture focusing the pelvis exhibiting left transient osteoporosis of hip

\section{Conclusion}

When an Indian woman in her last trimester of pregnancy experiences excruciating pain in her hip joints which is compromising her normal daily activities, she should be evaluated empathetically for TOH with bone marrow edema. Magnetic resonance imaging does support the diagnosis. Strict bed rest, analgesics, and bisphosphonates therapy will cure the disease fully.

We confirm that our Indian pregnant women can face the challenge of this rare condition of $\mathrm{TOH}$ with bone marrow edema which can be treated well conservatively.

\section{Ethical Approval}

This article does not contain any studies trial with human participants or animals performed by any of the authors.

\section{References}

1. Suresh SS, Thomas JK, Raniga S. Migrating transient osteoporosis of the hip in a 30 year old man. Indian J Orthop 2009;43(3):301-304. DOI: 10.4103/0019-5413.50872.

2. Abdulhak AAB, Ba-Mougadam FA, Al-Nakshabandi NA, et al. Transient osteoporosis of the hip/bone marrow edema syndrome with soft tissue involvement: a case report. Oman Med J 2011;26(25):353-355. DOI: 10.5001/omj.2011.86.

3. Joshi M. Transient osteoporosis of the hip: a differential diagnosis which is not to be Missed. J Musculoskeletal Pain 2014;22(4):392-395. DOI: 10.3109/10582452.2014.933995.

4. Choudhary MM, Ramesh J, Gupta S. Transient osteoporosis of hip. J Orthop Allied Sci 2015;3(1):44-47. DOI: 10.4103/2319-2585. 155929.

5. Hadji P, Boekhoff J, Hahn M, et al. Pregnancy—associated transient osteoporosis of the hip: results of case-control study. Arch Osteopors 2017;12(1):11. DOI: 10.1007/s11657-017-0310-y.

6. Vaishya R, Kumar A, Kumar V, et al. Transient osteoporosis of the hip: a mysterious cause of hip pain in adults. Indian J Orthop 2017;51(4):455-460. DOI: 10.4103/ortho.IJOrtho_607_16.

7. Dev R. Transient osteoporosis of hip presenting as stress fracture. Int J Anat Radiol Surg 2018;7(4):RC04-RC06.

8. Pallavi P, Padma S, Vanitha Anna Selvi D. Transient osteoporosis of hip and lumbar spine in pregnancy. J Obstet Gynaecol India 2012;62(S1):S8-S9. DOI: 10.1007/s13224-013-0355-9. 